Extension to the right heart chambers can cause valve obstruction, leading to cardiac insufficiency and death. Magnetic resonance and computed tomographic angiography are the most sensitive methods to diagnose this pathology and to enable planning of the most appropriate therapeutic strategy. ${ }^{2}$ Treatment consists in a total hysterectomy and bilateral salpingo-oophorectomy, along with removal of as much of the extrauterine tumor as possible. Recurrence can result from incomplete excision and may necessitate further surgical treatment. Because of the presence of estrogen receptors, antiestrogens such as tamoxifen may be given when the tumor is unresectable or to control for incomplete resection or recurrence of the tumor. $^{3}$
In conclusion, intravenous leiomyomatosis should be considered in the differential diagnosis of a middle-aged or older woman with a right-heart tumor. Histopathologic diagnosis must be made because other tumors, such as leiomyosarcomas of the retroperitoneum, may mimic intravascular leiomyomatosis.

\section{References}

1. Politzer F, Kronzon I, Wieczorek R, Feiner H, De Marco LE, Weintraub PR, et al Intracardiac leiomyomatosis: diagnosis and treatment. J Am Coll Cardiol. 1984;4: 629-34.

2. Maraj S, Pressman GS, Figueredo VM. Primary cardiac tumors. Int J Cardiol. 2009;133:152-6.

3. Tierney WM, Ehrlich CE, Bailey JC, King RD, Roth LM, Wann LS. Intravenous leiomyomatosis of the uterus with extension into the heart. Am J Med. 1980;69:471-5.

\title{
Management of postdissection thoracoabdominal aneurysm after previous frozen elephant trunk procedure with the E-vita Open Plus stent-graft
}

\author{
Vito Giovanni Ruggieri, MD, Amedeo Anselmi, MD, Issam Abouliatim, MD, PhD, and \\ Jean-Philippe Verhoye, MD, PhD, Rennes, France
}

The use of a hybrid stent-graft enables the extension of the aortic repair into the dissected descending aorta in patients undergoing aortic surgery through a median sternotomy. The frozen elephant trunk technique has been reported to decrease the rate of aortic reoperation, both open and endovascular, after repair of type A acute aortic dissection. ${ }^{1,2}$ Here we discuss a case of thoracoabdominal aneurysm treated with conventional surgery after a previous frozen elephant trunk procedure with the E-vita Open Plus hybrid endoprosthesis (JOTEC GmbH, Hechingen, Germany).

\section{CLINICAL SUMMARY}

A 33-year-old male patient was admitted with diagnosis of type A acute aortic dissection. The intimal tear was

\footnotetext{
From the Division of Cardiovascular and Thoracic Surgery, Pontchaillou Hospital, Rennes, France.

Disclosures: Authors have nothing to disclose with regard to commercial support. E-vita Open Plus is manufactured by JOTEC GmbH, Hechingen, Germany.

Received for publication Jan 26, 2012; revisions received March 6, 2012; accepted for publication March 16, 2012; available ahead of print April 16, 2012.

Address for reprints: Vito Giovanni Ruggieri, MD, Division of Cardiovascular and

Thoracic Surgery, Centre Hospitalier Universitaire Pontchaillou, 2 Rue Henri le

Guilloux, 35003 Rennes cedex 9, France (E-mail: gianvito_ruggieri@libero.it).

J Thorac Cardiovasc Surg 2012;144:e5-7

$0022-5223 / \$ 36.00$

Copyright (C) 2012 by The American Association for Thoracic Surgery

doi:10.1016/j.jtcvs.2012.03.031
}

localized into the ascending aorta. We performed an emergency David procedure, and the aortic arch was free from intimal tears. The day after surgery, the patient's primary care physician informed us about a diagnosis of Marfan syndrome, which was later confirmed histopathologically. Predischarge computed tomographic scan showed the extension of the dissection down to the abdominal aorta, without aneurysmal dilatation. Twenty-six months later, the patient came back with both worsening dyspnea and dorsal and lumbar pain. Computed tomographic scan revealed aneurysmal dilatation of the aortic arch (maximum diameter, $43 \mathrm{~mm}$ ), of the descending aorta $(56 \mathrm{~mm})$, and of the abdominal aorta $(66 \mathrm{~mm})$. The main visceral vessels emerged from the true lumen. Echocardiography revealed severe mitral regurgitation and moderate systolic dysfunction of the left ventricle. We performed a mitral valve replacement associated with aortic arch replacement and descending aortic stent-grafting according to the frozen elephant trunk technique ${ }^{3}$ with a 24-mm E-vita Open Plus hybrid prosthesis. Three months later, the patient was readmitted for intractable dorsal and lumbar pain. Computed tomographic scan showed complete thrombosis of the false lumen at the stent-graft level and partial thrombosis below (Figure 1). Nevertheless, the thoracoabdominal aortic diameter had increased. We decided to perform regular surgery for treatment of the thoracoabdominal aneurysm. Femorofemoral 


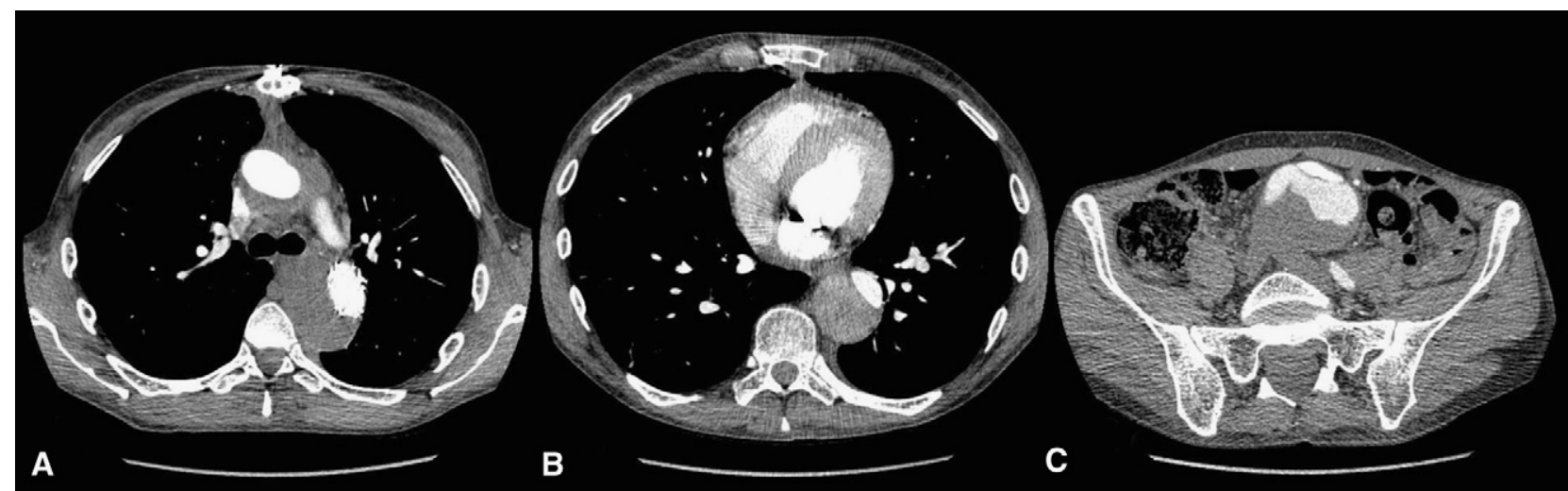

FIGURE 1. Transverse section contrast-enhanced computed tomographic scan control after placement of hybrid endoprosthesis. A, Complete thrombosis of the false lumen at the stent-graft level. B, Partial thrombosis at the lower descending aortic level. C, Patent false lumen at the abdominal level.

cardiopulmonary bypass was established. Through a left thoracophrenolaparotomy and under conditions of hypothermic circulatory arrest, the aneurysm was opened. The distal portion of the E-vita Open Plus stent-graft was therefore resected. A 24-mm Dacron polyester fabric tube graft was sutured to the stent-graft and crossclamped to restart cardiopulmonary bypass from the side branch of the tube. Both patent intercostal arteries and visceral patch were reimplanted on the graft. The intercostal arteries were reimplanted with an aortic patch directly anastomosed to the

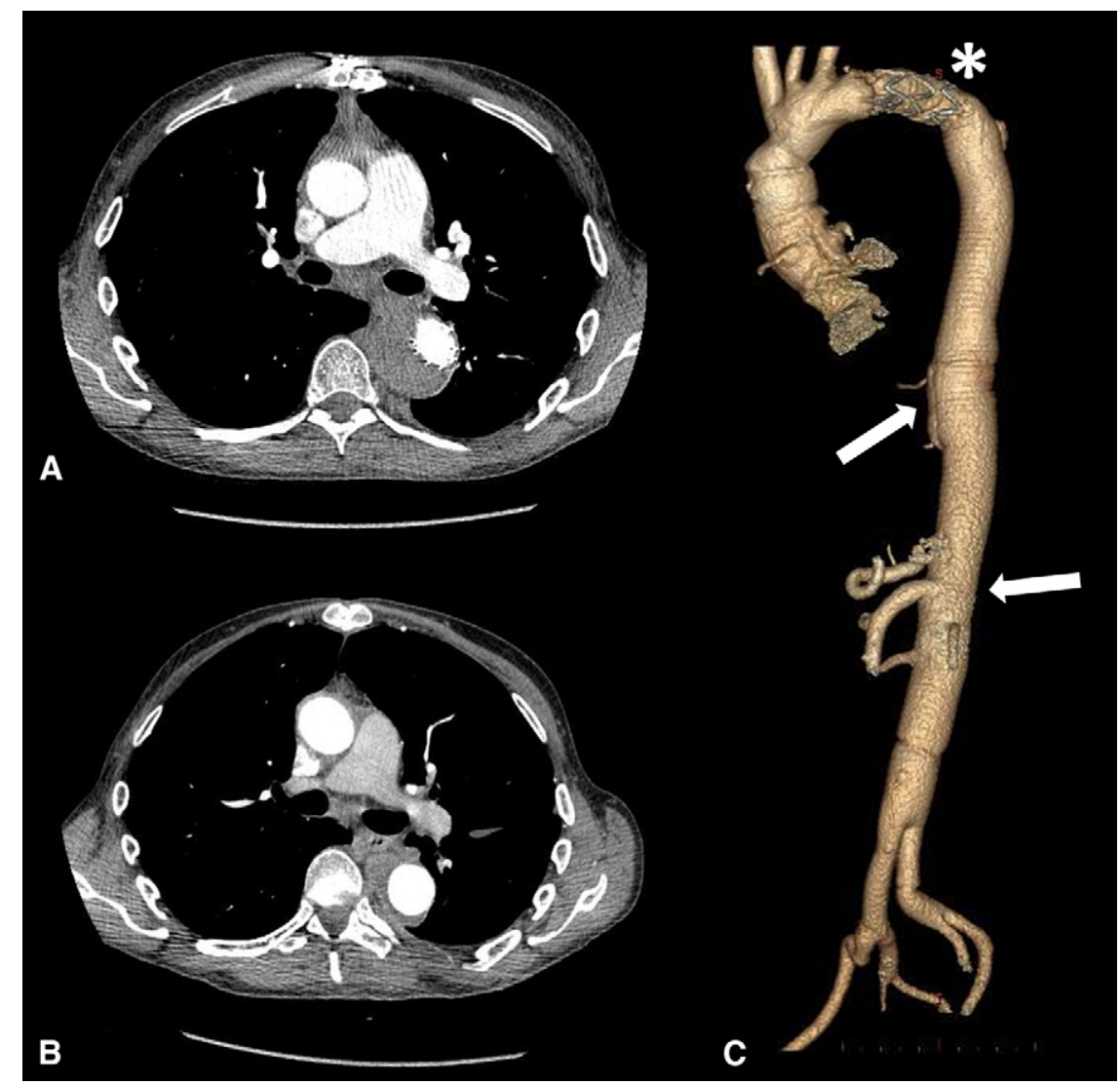

FIGURE 2. Transverse section contrast-enhanced computed tomographic scans at the level of the bifurcation of the main pulmonary artery, showing the entire E-vita Open Plus (JOTEC GmbH, Hechingen, Germany) endoprosthesis (A) and the tube graft after endoprosthesis resection (B). C, Threedimensional reconstruction of the aorta showing the residual segment of stent-graft and its anastomosis with the tube graft (white asterisk) and the prosthetic replacement of the entire aorta with reimplantation of the segmental and of the visceral arteries (white arrows). 
tube. The abdominal aorta was replaced down to the iliac junction. No postoperative complications were noted. Predischarge computed tomographic scan showed excellent result of thoracoabdominal aorta replacement (Figure 2).

\section{DISCUSSION}

A multicenter investigation of the hybrid endoprosthesis for obliteration of the false lumen in the downstream thoracic aorta has reported the feasibility and efficacy of this approach, with a $25 \%$ rate of distal aortic reintervention at midterm follow-up. ${ }^{4}$ The published reports scarcely discuss the optimal management for further intervention on the downstream aorta. The connective disorder of our patient was likely a determinant of subsequent aortic enlargement, despite successful positioning of hybrid endoprosthesis and even though we did not oversize the prosthesis more than $10 \%$ as recommended. ${ }^{4}$ Moreover, the frequency of patients with Marfan syndrome in the published series is low and does not allow us to draw definitive conclusions regarding the reliability of endoprostheses in this subgroup. Finally, the absence of a distal landing zone before the origin of the visceral vessels and the risk of spinal cord injury ${ }^{5}$ oriented our strategy toward open surgery.

During the thoracoabdominal operation, we unexpectedly localized 3 couples of patent intercostal arteries at the T8 through T10 levels, just near the distal landing zone of the E-vita Open Plus endoprosthesis. The proximal part of the descending aorta was managed under hypothermic circulatory arrest to avoid crossclamping of the stentgraft. On the basis of our institutional expertise with the performance of hypothermic circulatory arrest, we decided to adopt this strategy instead of using a balloon occluder to manage the proximal descending aorta. The distal portion of the stent-graft was resected, and a confident end-to-end anastomosis was performed between the stent-graft stump and a Dacron polyester fabric tube. The stent struts did not at any time present an obstacle to the realization of the anastomosis.

In conclusion, the surgeon should consider the possibility of resecting a portion of the distal stent-graft of the E-vita Open Plus endoprosthesis at the time of open surgery on the thoracoabdominal aorta to perform the proximal anastomosis at the desired level. This may allow a more radical resection of the diseased aortic tissue and the reimplantation of all patent intercostal arteries.

\section{References}

1. Tsagakis K, Pacini D, Di Bartolomeo R, Gorlitzer M, Weiss G, Grabenwoger M, et al. Multicenter early experience with extended aortic repair in acute aortic dissection: is simultaneous descending stent grafting justified? J Thorac Cardiovasc Surg. 2010;140:S116-20; discussion S142-6.

2. Tsagakis K, Pacini D, Di Bartolomeo R, Benedik J, Cerny S, Gorlitzer M, et al. Arch replacement and downstream stent grafting in complex aortic dissection: first results of an International registry. Eur J Cardiothorac Surg. 2011;39: 87-94.

3. Di Bartolomeo R, Di Marco L, Armaro A, Marsilli D, Leone A, Pilato E, Pacini D. Treatment of complex disease of the thoracic aorta: the frozen elephant trunk technique with the E-vita open prosthesis. Eur J Cardiothorac Surg. 2009; 35:671-6.

4. Pacini D, Tsagakis K, Jakob H, Mestres CA, Armaro A, Weiss G, et al. The frozen elephant trunk for the treatment of chronic dissection of the thoracic aorta: a multicenter experience. Ann Thorac Surg. 2011;92:1663-70.

5. Feezor R, Martin T, Hess PJ Jr, Daniels MJ, Beaver TM, Klodell CT, et al. Extent of aortic coverage and incidence of spinal cord ischemia after thoracic endovascular aneurysm repair. Ann Thorac Surg. 2008;86:1809-14.

\title{
A left atrial myxomalike rhabdomyosarcoma
}

\author{
Domenico Corradi, MD, ${ }^{\mathrm{a}}$ Giovanni Andrea Contini, MD,${ }^{\mathrm{b}}$ Tiziano Gherli, MD, ${ }^{\mathrm{b}}$ and \\ Francesco Nicolini, MD, PhD, ${ }^{\mathrm{b}}$ Parma, Italy
}

Cardiac tumors are exceedingly rare, with their frequency being estimated between $0.0017 \%$ and $0.33 \%{ }^{1}$ Despite its limited myocardial mass, the left atrium is

\footnotetext{
From the Section of Pathology, ${ }^{\mathrm{a}}$ Department of Pathology and Laboratory Medicine, and the Heart Surgery Section, ${ }^{\mathrm{b}}$ University of Parma, Parma, Italy.

Disclosures: Authors have nothing to disclose with regard to commercial support.

Received for publication March 18, 2012; accepted for publication March 30, 2012; available ahead of print May 2, 2012.

Address for reprints: Domenico Corradi, MD, Section of Pathology, Department of Pathology and Laboratory Medicine, University of Parma, Via Gramsci 14, 43126 Parma, Italy (E-mail: domenico.corradi@unipr.it).

J Thorac Cardiovasc Surg 2012;144:e7-10

0022-5223/\$36.00

Copyright (c) 2012 by The American Association for Thoracic Surgery

doi:10.1016/j.jtcvs.2012.03.073
}

very frequently involved in both benign and malignant primary cardiac tumors, with polypoid myxoma being the most frequent type. ${ }^{2}$ We report here the exceptional case of a fully polypoid left atrial rhabdomyosarcoma without myocardial infiltration, mimicking a cardiac myxoma.

\section{CLINICAL SUMMARY}

A 39-year-old man was referred to our heart surgery unit because of the recent discovery of a left atrial mass, echocardiographically consistent with cardiac myxoma. The patient's recent medical history had been characterized by such nonspecific symptoms as sporadic fever, 